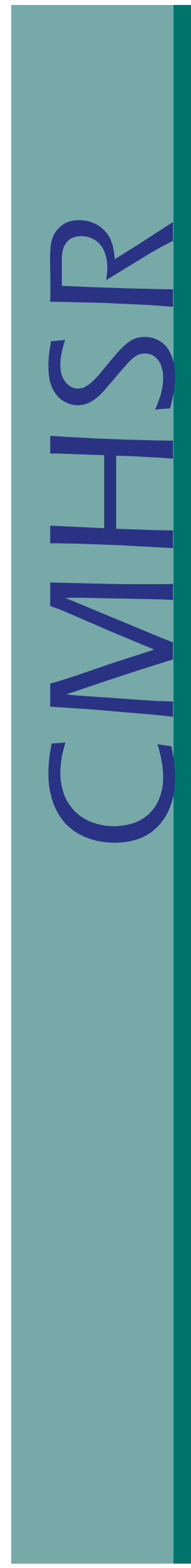

\title{
Families with Overlapping Needs
}

Betsy Hinden, Ph.D., Bernice Gershenson, M.P.H., Valerie Williams, M.A., M.S., and Joanne Nicholson, Ph.D.

$\mathrm{O}$ ver the course of their lifetime, the majority of American adults who meet criteria for mental illness are parents. ${ }^{1}$ Addressing the needs of families in which a parent has a mental illness is essential for such a family's well-being and functioning. Parents with mental illness face all the challenges of other adults trying to balance their roles as spouses, parents and workers. However, due to their symptoms, both their overall functioning and parenting ability may be adversely affected. Resolving this issue requires significant changes in the way current interventions and services are delivered.

\section{Introduction}

Since the early 1990's, the Substance Abuse and Mental Health Service Administration has supported the development of children's systems of care (SOCs) to address the needs of those children with the most serious emotional and behavioral disorders. SOCs focus on providing a comprehensive array of integrated, family-driven, community-based services $^{2}$ and rely heavily on parent involvement in care planning and implementation. Mental illness and substance abuse issues may affect a family's ability to participate effectively within SOCs. Identifying children with a family history of mental illness and/or substance abuse is therefore essential to making SOCs fully accessible to and effective for all participating families

From 2004-2005, the Center for Mental Health Services Research conducted secondary analysis utilizing data from the Descriptive Study of Phases II and III of the National Evaluation Outcomes Study (as of December 2003) of the Comprehensive Community Mental Health Services for Children and Families Program. ${ }^{2}$

The aims of the study were to:

- Examine the prevalence of family mental illness and family substance abuse among children entering SOCs;

- Compare families with histories of mental illness and substance abuse to families without these histories, with respect to child and family risk factors at the time of enrollment into the program; and

(C) 2006 Center for Mental Health Services Research Department of Psychiatry

University of Massachusetts Medical School

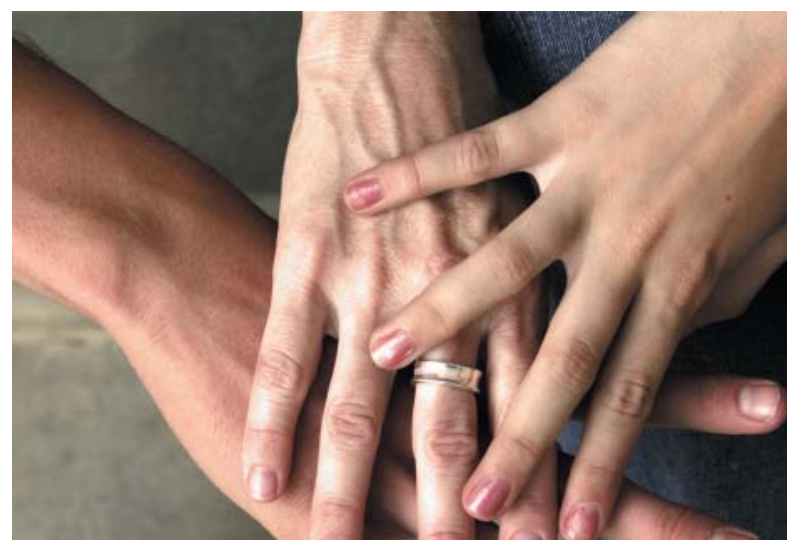

- Illuminate related implications for research, policy and practice.

\section{Methods}

The dataset analyzed contained information on all children and families entering SOC's in 90 communities within the US and territories. Only respondents who were biological parents were included in the current study ( $n=8,782$ children). Four groups were distinguished with respect to family history of mental illness (MI) and substance abuse (SA): Neither group (no history of MI or SA; $n=2,347$ ); MI Only group (history of MI, no history of SA; $n=1,348$ ); SA Only group (history of SA, no history of MI; $n=1,879$ ); Co-occurring group (co-occurring histories of MI and SA; $n=3,208$ ).

\section{Results}

Fifty-two percent of participating families reported a history of mental illness in the biological family and $58 \%$ reported a history of substance abuse. Over a third $(36.5 \%)$ of the sample reported a history of both mental illness and substance abuse. As can be seen in Table 1, family histories of MI and/or SA were associated with significantly greater risk for all child and family variables examined. In addition, certain risks were associated more strongly with either mental illness or substance abuse. For example, family history of MI in the absence of SA was associated with greater risk of psychiatric hospitalization and suicide attempts, while family history of SA in the absence of MI was associated with greater risk of child history of substance abuse. Children and caregivers from families with co-occurring histories of MI and SA clearly exhibited the greatest risk overall, as they experienced the cumulative risks associated with both family history of MI and SA. Two limitations 
Table 1. Child and Family Trauma and Child Psychiatric History: Percent Reporting by Group

\begin{tabular}{|c|c|c|c|c|c|}
\hline \multirow[b]{2}{*}{ Child and Family Trauma History } & \multicolumn{4}{|c|}{ Family History Group } & \multirow{2}{*}{$\begin{array}{l}\text { Model p } \\
\text { Value }\end{array}$} \\
\hline & Neither & MI Only & SA Only & Co-occurring & \\
\hline Child ever physically abused & $10 \%$ & $18 \%$ * & $20 \%$ * & $30 \%$ * & $<.0001$ \\
\hline Child ever sexually abused & $12 \%$ & $16 \% \wedge$ & $17 \% \dagger$ & $23 \%^{*}$ & $<.0001$ \\
\hline $\begin{array}{l}\text { History of domestic violence in child's biological } \\
\text { family (but child was not the direct target of the violence) }\end{array}$ & $22 \%$ & $38 \%{ }^{*}$ & $53 \%{ }^{*}$ & $67 \%{ }^{*}$ & $<.0001$ \\
\hline Biological parent convicted of a crime & $15 \%$ & $24 \%{ }^{*}$ & $52 \%{ }^{*}$ & $61 \%{ }^{*}$ & $<.0001$ \\
\hline \multicolumn{6}{|l|}{ Child Psychiatric History } \\
\hline Child ever had a previous psychiatric hospitalization & $18 \%$ & $33 \%{ }^{*}$ & $20 \%$ & $33 \%^{*}$ & $<.0001$ \\
\hline Child ever attempted suicide & $10 \%$ & $15 \%{ }^{*}$ & $12 \%$ & $19 \%{ }^{*}$ & $<.0001$ \\
\hline Child ever been sexually abusive to others & $3 \%$ & $6 \% \dagger$ & $5 \% \wedge$ & $8 \%{ }^{*}$ & $<.0001$ \\
\hline $\begin{array}{l}\text { Child ever ran away without his/her caregiver knowing } \\
\text { where he/she was }\end{array}$ & $22 \%$ & $29 \%$ * & $32 \%{ }^{*}$ & $35 \%{ }^{*}$ & $<.0001$ \\
\hline Child have a history of substance abuse & $12 \%$ & $13 \%$ & $26 \%{ }^{*}$ & $23 \%{ }^{*}$ & $<.0001$ \\
\hline
\end{tabular}

Child demographic variables age, race, gender, parent education and household income were controlled in all analyses.

Significant differences between the Neither group and each of the other groups are indicated by the following * $p<.001 ; \dagger p<.01 ; \wedge p<.05$.

of this study are important to note. First, the cross-sectional nature of the study makes it impossible to disentangle the timing and relationships among variables examined. Second, because this was a secondary analysis, the factors we could investigate were limited to questions asked previously. Specifically, there were no data available for the number of parents, as distinguished from family members, affected by mental illness or substance abuse.

\section{Policy and Practice Implications and Recommendations}

These findings have important implications at both the systems and family levels including the following:

- Families entering SOCs reflect important differences that are likely to affect their experience in SOCs as well as their outcomes;

- There are high rates of families entering SOCs with overlapping child and parent mental health issues and trauma histories that are likely to affect their specific service needs as well as outcomes; and

- Family-centered approaches that take these needs into account are critical to effective SOCs.

Family-centered services that address the strengths and needs of all family members require transformation at both the system and provider levels. To effectively support family centered approaches, within SOCs, this study suggests:

- Integrating adult and child service systems;

- Strong collaboration and cross training between adult and child service agencies across service sectors;

- Educating the state mental health authorities on the latest research regarding the needs of families with mental health and substance abuse problems;
- Incorporating trauma-specific and trauma-informed services into service plans for adults and children;

- Educating providers about the experiences of parents and children affected by family mental illness and/or substance abuse;

- Educating providers and administrators on the issues of custody loss, stigma faced by parents coping with mental health problems, and alternative custody arrangements; and

- Developing appropriate funding mechanisms to support familycentered initiatives.

\section{References}

1. Nicholson, J., Biebel, K., Katz-Leavy, J., \& Williams, V. (2003). The prevalence of parenthood in adults with mental illness: Implications for state and federal policy makers, programs and providers. In R.W. Manderscheid \& M. J. Henderson (Eds.). Mental Health United States, (2002). Rockville, MD: U.S. Department of Health and Human Services, Substance Abuse and Mental Health Services Administration, Center for Mental Health Services.

2. Center for Mental Health Services (2004). The Comprehensive Community Mental Health Services for Children and Their Families Program: Evaluation findings-Annual report to Congress, 2004. Atlanta, GA: ORC Macro.

Visit us on-line at www.umassmed.edu/cmhsr 